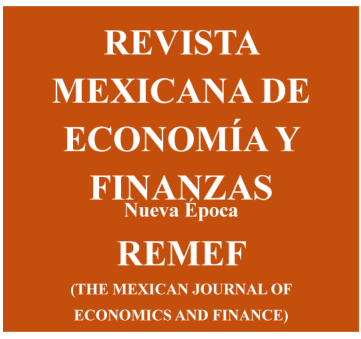

\begin{tabular}{c}
\hline Revista Mexicana de Economía y Finanzas \\
Nueva Época \\
Volumen 15 Número 2, Abril - Junio 2020, pp. 263-276 \\
DOI: https://doi.org/10.21919/remef .v15i2.378 \\
(Primera recepción: 18/febrero/2019 \\
última recepción: $27 /$ enero/2020, \\
aceptado: $17 /$ febrero/2020)
\end{tabular}

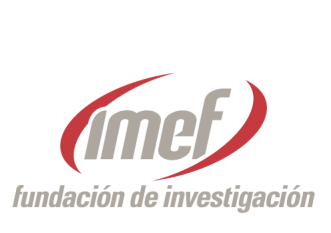

\title{
Análisis técnico y financiero del potencial energético del viento para generar energía eléctrica: el caso de Apan, Hidalgo
}

\author{
Tomás Gómez Rodríguez ${ }^{1}$ \\ Universidad Autónoma del Estado de Hidalgo, México \\ Adriana Cortazar Martínez \\ Universidad Autónoma del Estado de Hidalgo, México \\ Adriana Zambrano Reyes \\ Universidad Autónoma del Estado de Hidalgo, México
}

\section{Resumen}

Objetivo: Evaluar el potencial energético del viento en Apan, Hidalgo. Metodología: Se emplean dos tipos de análisis: el análisis técnico y el análisis financiero. Para el estudio técnico se emplea la función de densidad de probabilidad Weibull. Así mismo, para el análisis financiero se evalúan los siguientes parámetros: valor presente neto, la tasa interna de rendimiento, el Costo Nivelado de la Electricidad, el período de recuperación y la razón costo/beneficio. Resultados: Se encontró que de los doce aerogeneradores considerados solo ocho son técnicamente viables y ninguno económicamente. Recomendaciones: Se recomienda no hacer la instalación de aerogeneradores comerciales. Limitaciones e implicaciones: La limitación de la función de densidad de probabilidad de Weibull es que no representa correctamente las probabilidades de la velocidad del viento cuando esta es muy baja o cercana a cero. Orignalidad: De acuerdo a la revisión de la literatura este estudio es la primera investigación para el caso de Apan, Hidalgo que analiza el potencial energético del viento y su factibilidad financiera. Conclusiones: La evidencia sugiere que el potencial del viento es adecuado para aplicaciones que no están conectadas a la red eléctrica e insuficiente para la producción de energía a escala comercial.

Clasificación JEL: Q42, Q47, Q56

Palabras claves: Fuentes alternativas de energía, pronóstico de energía, sustentabilidad

\section{Technical and Financial Analysis of the Wind Energy Potential to Generate Electric Power: the Case of Apan, Hidalgo}

\section{Abstract}

Objective: Evaluate the energy potential of wind in Apan, Hidalgo. Methodology: Two types of analysis were used: technical analysis and financial analysis. The Weibull probability density function is used for the technical study. Likewise, the following parameters are evaluated for the financial analysis: net present value, the internal rate of return, the Levelized Cost of Electricity, the recovery period and the cost / benefit ratio. Results: It was found that of the twelve wind turbines considered only eight are technically viable and none economically. Recommendations: It is recommended not to install commercial wind turbines. Limitations and implications: The main limitation of Weibull density function is that it does not correctly represent the probabilities of

\footnotetext{
${ }^{1}$ Correspondencia: Tomás Gómez Rodríguez. Dirección: Carretera Apan-Calpulalpan Km.8, Col.Chimalpa, C. P. 43920 Apan, Hgo. Teléfono: 017717172000 extensión 5806. Correo electrónico: tomas ${ }_{g}$ omez@uaeh.edu.mx.
} 


\section{Abstract}

wind speed when it is very low or near zero. Originality: According to the literature review, this study is the first investigation in the case of Apan, Hidalgo, which analyzes the wind's energy potential and its financial feasibility. Conclusions: The evidence suggests that the potential of the wind is suitable for applications that are not connected to the electricity grid and insufficient for the production of energy on a commercial scale. JEL Classification: Q42, Q47, Q56

Keywords: Alternative energy sources, energy forecasting, sustainability

\section{Introducción}

El consumo mundial de energía primaria en 2016 fue de 13276.3 millones de toneladas equivalentes de petróleo, siendo las principales fuentes de energía: el petróleo con $33 \%$, el carbón con $28 \%$ y el gas natural con $24 \%$. Por otro lado, solo el $3 \%$ de la energía primaria a nivel mundial proviene de fuentes renovables (BP 2017). El aumento de la demanda de energía a nivel mundial será $48 \%$ mayor en el año 2040 de acuerdo a la agencia internacional de energía (EIA 2016). Una de las formas de energía más demandada alrededor del mundo es la energía eléctrica. La generación de energía eléctrica crecerá cerca de $69 \%$ para el año 2040. Los combustibles fósiles en la actualidad representan un $67 \%$ del total de la generación de electricidad, mientras que la energía renovable representa solo un $22 \%$. En México el $57 \%$ de la energía eléctrica se genera empleando gas natural, el $11.1 \%$ utiliza carbón como fuente de energía y el $8.8 \%$ utiliza combustibles fósiles mientras que solo el $3 \%$ de la energía eléctrica en México proviene de fuentes renovables (SENER 2015).

El uso de combustibles fósiles para producir energía ha generado preocupación debido a temas como: reservas finitas, impactos ambientales como: el cambio climático, lluvia acida y calentamiento global, incrementos en la demanda de electricidad e incrementos en su costo (Dabbaghiyan et al. 2016). Lo que vuelve extremadamente difícil para países pobres y en desarrollo mantener un suministro adecuado de energía. Para poder superar este problema se explora el empleo de fuentes de energía renovables, las cuales son alternativas a los combustibles fósiles. Las principales ventajas de las fuentes de energía renovables de acuerdo al trabajo de Alamdari et al. (2012) son: sustentabilidad, se pueden implementar en cualquier lugar y son amigables con el medio ambiente.

El viento es originado debido a las diferencias del flujo natural del aire a través del mar o sobre la superficie de la tierra. La rotación de la tierra y las diferencias de temperatura son la causa del calentamiento y enfriamiento desigual de las distintas superficies de la tierra lo que causa que sople el viento. El calor proveniente del sol es recibido por áreas de agua y tierra en diferentes cantidades, como resultado, el aire caliente asciende y el aire frío lo reemplaza causando vientos locales de acuerdo a Ellabban et al. (2014).

El viento se convierte en energía a través del empleo de aerogeneradores, haciendo de la energía eólica una de las fuentes de energía renovable más importante de los últimos tiempos, debido a sus ventajas. Entre estas ventajas están: que es limpia, abundante, relativamente barata, interminable y ambientalmente benigna (Akpinar 2013). La investigación en esta área se centra en mejorar el conocimiento y las tecnologías para generar energía eólica. El uso de la tecnología del aerogenerador ha recibido mayor atención y se ha desarrollado rápidamente en las últimas décadas. La energía eólica es una de las fuentes de energía renovable más eficiente, pero requiere un análisis muy detallado de las características del viento de la región seleccionada. Las características del viento como la función de densidad de probabilidad de la velocidad del viento y su dirección pueden tener grandes diferencias entre diferentes regiones aun cuando estas regiones se encuentren cerca.

Una investigación completa de los datos de la velocidad, densidad y dirección del viento es de la mayor importancia para obtener estimados confiables del potencial del viento. Un requisito necesario para la inves- 
tigación del potencial del viento en cualquier locación, es una función de distribución estadística adecuada para modelar los datos de la velocidad del viento de acuerdo al trabajo de Aien et al. (2014).

Existe una amplia gama de funciones de distribución y la calidad del análisis de la velocidad del viento depende de la capacidad de la función de densidad de probabilidad para describir la distribución de frecuencia observada. Aunque existen muchos modelos potenciales de probabilidad para el análisis de la distribución, la distribución Weibull es una de las más usada, además de que es ampliamente aceptada y citada en la literatura del viento. Se considera un enfoque estándar debido a su precisión, simplicidad y flexibilidad de acuerdo a Fazelpour et al. (2017). Dicha función de distribución está caracterizada por dos parámetros: forma y escala. El parámetro de escala controla el rango mientras que el parámetro de forma define la amplitud de la distribución de los datos. Una distribución más amplia implica un parámetro de forma más pequeña y menor será el valor máximo de la distribución.

México cuenta con un importante potencial de generación de energía eléctrica a través del sol, el viento y la biomasa. Específicamente el potencial estimado es de 16351 gigawatt hora por año (GWh/año) para el sol, $19805 \mathrm{GWh} /$ año para el viento y $2396 \mathrm{GWh} /$ año de acuerdo a Pérez-Denicia et al (2017). En México en el año 2016 se encontraban operando 1935 aerogeneradores con una capacidad de 3527 megawatts (MW) en 42 parques eólicos. Y para el cierre de 2017 se habían instalado 4005 MW (AMDEE 2018). Los parques eólicos con mayor capacidad instalada hasta el año 2015 son operados por: Eurus S.A. de C.V. con una capacidad instalada de 250.52 MW el parque se ubica en Juchitán de Zaragoza, Oaxaca, Fuerza y Energía BII HIOXO, S.A. de C.V. con una capacidad instalada de 226.8MW el parque se ubica en Juchitán de Zaragoza, Oaxaca y Dominica Energía Limpia S.A. de C.V. con una capacidad instalada de 200MW el parque se ubica en Charcas, San Luis Potosí de acuerdo a Pérez-Denicia et al. (2017).

Las áreas de la república mexicana con mayor potencial de viento son la península de Baja California, la región central, la costa del golfo, la península de Yucatán y el Istmo de Tehuantepec de acuerdo a CancinoSolórzano et al. (2011). Apan, Hidalgo se encuentra ubicado en la región central de México que de acuerdo a Hernandez Escobedo et al. (2010) puede llegar a generar hasta 10000 kilowatt (kW) por aerogenerador instalado por año.

Algunos trabajos que se han realizado sobre los prospectos y el estado de la energía eólica en México son los de: Magar et al. (2018) ellos realizan un análisis técnico-económico con restricciones socio-ecológicas para la explotación del viento mediante parques eólicos fuera de la costa. La región de estudio se localiza en el Golfo de California, entre Santa Rosalía-Guaymas y el delta del río Colorado. Proponen una capacidad técnica instalada de $131 \mathrm{GW}$ empleando aerogeneradores con una capacidad de $6 \mathrm{MW}$, cuando se considera el uso de las zonas de pesca, y de $52 \mathrm{GW}$ cuando se toman en cuenta restricciones sociales y ecológicas. La cifra de 52 GW es equivalente al $84 \%$ de la producción de energía en México. Hernández Escobedo et al. (2014) estudian el caso de cuatro estados del noroeste de México, encuentran que para la mayor parte de estos estados se observa una velocidad del viento por encima de los $4.51 \mathrm{~m} / \mathrm{s}$, Hernández Escobedo et al. (2011) encuentran que para explotar el potencial del viento se debe considerar una velocidad del viento mínima entre las 8:00 y 14:00 h. y una velocidad máxima cerca de las 24:00 h. En el trabajo de Cancino-Solórzano et al. (2011) encuentran que el área con el mayor potencial del viento es el estado de Oaxaca y se localizan en la parte sur del Istmo de Tehuantepec donde la velocidad se observa en un rango de 7.3 a $7.7 \mathrm{~m} / \mathrm{s}$, mientras en las montañas del istmo la velocidad del viento es mayor a $8.5 \mathrm{~m} / \mathrm{s}$. En el trabajo de Hernández-Escobedo et al. (2010) encuentran que México tiene un potencial de más de 1700 h útiles del viento por año en prácticamente todo el país con la excepción del estado de Chiapas. En los estados de Puebla, Hidalgo, Zacatecas, Tlaxcala y Estado de México, existen áreas que pueden generar hasta $10000 \mathrm{~kW}$, por aerogenerador instalado. Por otro lado, la península de Yucatán podría generar hasta 15000kW.

El objetivo es evaluar técnica y financieramente el potencial energético del viento en Apan, Hidalgo con el 
fin de generar energía eléctrica. Este estudio se concentra en la determinación de la probable producción de energía eólica para evaluar la factibilidad técnica, con este fin se hace uso de la distribución Weibull. Mientras que para la factibilidad financiera se consideran los siguientes parámetros financieras: valor presente neto, la tasa interna de rendimiento, el costo nivelado de la electricidad, el período de recuperación y la razón costo/beneficio.

\section{Zona de estudio}

La región de Apan Hidalgo está ubicada en las coordenadas 19 42' latitud norte, 98 27' latitud oeste, a una altura de 2480 metros sobre el nivel del mar. El $90 \%$ de su extensión es llanura y el restante $10 \%$ es lomerío.

Se revisaron los datos históricos de las variables climáticas del Municipio de Apan, Se utilizaron los datos climatológicos obtenidos de la Estación Meteorológica Automática (EMA)-Apan del Servicio Meteorológico Nacional (SMN). Se analizaron en total 17665 registros para generar una base de datos con la que se trabajó para todos los cálculos.

\section{Metodología}

\subsection{Análisis técnico}

Para evaluar el potencial del viento para la producción de electricidad en Apan, Hidalgo es necesario como primer paso aplicar la ley exponencial de Hellman a los datos de la estación meteorológica para obtener datos de la velocidad del viento para diferentes alturas. Los datos obtenidos de la estación meteorológica se ajustan a la distribución de Weibull y se estiman los parámetros de escala y forma, así como la función de densidad de probabilidad. Una vez obtenida esta información podemos calcular la: velocidad del viento más probable, la velocidad del viento que conlleva la máxima energía, la producción de energía anual por aerogenerador y por último el factor capacidad por aerogenerador.

\section{Perfil del viento}

La velocidad promedio del viento como una función de la altura por encima del suelo permite encontrar el perfil del viento del lugar a investigar. Por razones de costo eficiencia; la altura de la torre de medición está por debajo de la altura de instalación de los aerogeneradores modernos. Por lo tanto, las velocidades del viento deben ser extrapoladas basándose en el perfil del viento. Un método común para extrapolar la velocidad del viento a diferentes alturas es la ley exponencial de Hellman o ley de poder; definida como en Manwell et al. (2012):

$$
v_{2}=v_{1} \cdot\left(\frac{h_{2}}{h_{1}}\right)^{\alpha}
$$

Donde $v_{2}$ es la velocidad del viento a la altura $h_{2}$ y $v_{1}$ es la velocidad del viento a la altura $h_{1}$, el exponente de Hellman es $\alpha$. Para calcular $\alpha$ se usa la siguiente ecuación:

$$
\alpha=\log \left(\frac{v_{2}}{v_{1}}\right) \log \left(\frac{h_{2}}{h_{1}}\right)
$$

Si solo se conoce la velocidad del viento para una altura, $\alpha$ debe ser estimada. $\alpha$ depende de varios temas, estos incluyen aspereza y tipo de terreno del lugar. 


\section{Función de densidad de probabilidad Weibull}

El potencial energético y las características del viento de un lugar específico pueden ser evaluados empleando un análisis estadístico de largo plazo de las observaciones meteorológicas. Existen diferentes funciones de probabilidad están son utilizadas para describir la frecuencia de la velocidad del viento en un período de tiempo, entre estas destacan las de: Weibull, Rayleigh, tres parámetros beta, lognormal, y gamma. Se emplea la función de distribución de Weibull debido a su precisión general. La principal limitación de la función de densidad de probabilidad Weibull es que no representa correctamente las probabilidades de la velocidad del viento cuando esta es muy baja o cercana a cero. Sin embargo, para estimar el potencial del viento para la generación de electricidad empleando aerogeneradores comerciales, generalmente no es necesario considerar las velocidades del viento muy bajas; por dos razones la primera es que la energía del viento a baja velocidad es pequeña (la energía del viento es proporcional al cubo de la velocidad del viento) y la segunda razón es que estas velocidades están por debajo del rango operativo de los aerogeneradores (la velocidad a la que empiezan a operar los aerogeneradores es generalmente de 2.5 a $3.5 \mathrm{~m} / \mathrm{s}$ ) (Fazelpour et al. 2017). La función de densidad de probabilidad de Weibull está dada por la siguiente ecuación de acuerdo a Stevens y Smulders (1979):

$$
f(U)=\left(\frac{k}{c}\right) \cdot\left(\frac{U}{c}\right)^{k-1} \exp \left[-\left(\frac{U}{c}\right)^{k}\right]
$$

Donde $f(U)$ denota la función de densidad probabilidad de la velocidad del viento $U, k$ es un parámetro de forma y $c$ es un factor de escala.

\section{Viento más probable}

La velocidad del viento más frecuente $U_{m p}$ es también la velocidad del viento más probable y se puede calcular con la siguiente ecuación de acuerdo al trabajo de Alamdari et al. (2012):

$$
U_{m p}=c\left(1-\frac{1}{k}\right)^{\frac{1}{k}}
$$

\section{Velocidad del viento que conlleva la máxima energía}

La velocidad del viento que produce la máxima cantidad de energía $U_{m e}$ en un año es otra medida importante en el diseño de los aerogeneradres y se obtiene de la siguiente forma de acuerdo al trabajo de Alamdari et al. (2012):

$$
U_{m e}=c\left(1+\frac{2}{k}\right)^{\frac{1}{k}}
$$

\section{Producción de energía anual}

Para un aerogenerador la producción de energía media se expresa de la siguiente manera como en Manwell et al. (2012):

$$
E=T \int_{U_{\text {in }}}^{U_{\text {out }}} f(U) p(U) d U
$$

Donde $f(U)$ es la función de densidad de probabilidad de la velocidad del viento $U, p(U)$ es la curva de potencia del aerogenerador (mide como cada aerogenerador convierte el viento disponible en energía) y $\mathrm{T}$ es el período de producción. La producción de energía empieza en la velocidad de corte $U_{i n}$ y termina en la velocidad de corte $U_{\text {out }}$. 
Por tanto, la producción de energía para cada aerogenerador $i$ se calcula de acuerdo a la siguiente expresión:

$$
A E P_{i}=\text { Aturb }_{i} \frac{\rho}{\rho_{p c}} H \sum_{b=1}^{n} W\left(U_{b}\right) P\left(U_{b}\right)
$$

Donde Aturb $_{i}$ es la disponibilidad promedio del aerogenerador i, $\rho$ es la densidad del aire del lugar, $\rho_{p c}$ es la densidad del aire para la curva de potencia. $W\left(U_{b}\right)$ es la probabilidad del segmento de la velocidad del viento $U_{b}$ estimada por la distribución de Weibull y $P\left(U_{b}\right)$ es la potencia producido por el segmento de la velocidad del viento. $\mathrm{H}$ es el número de horas de operación del aerogenerador.

\section{Factor de capacidad}

La productividad de un aerogenerador para un sitio puede ser evaluada por el factor de capacidad CF. El factor de capacidad se define como la proporción de la energía producida en un período dado, con respecto a la maxima cantidad de energía que se puede producir con un aerogenerador en particular. Entre más alto es el factor de capacidad, mayor es el rendimiento del aerogenerador. El factor de capacidad se calcula de la siguiente forma (Mohammadi y Mostafaeipour 2013):

$$
C F_{i}=\frac{A E P_{i}}{\operatorname{Prated}_{i}(8760)}
$$

Donde $C F_{i}$ es el factor de capacidad para el aerogenerador i, $A E P_{i}$ es la producción de energía anual de energía electrica por aerogenerador i, Prated $_{i}$ es la potencia declarada del aerogenerador i y el máximo de horas que puede operar un aerogenerador (8760 horas) en un año.

\subsection{Análisis financiero}

Los parámetros financieros generalmente utilizados para analizar la factibilidad financiera son: el valor presente neto, la tasa de rendimiento interno, el costo nivelado de la electricidad, el período de recuperación y la razón costo beneficio. Todos estos parámetros se discuten y son estimados para todos los aerogeneradores seleccionados.

\section{Valor Presente Neto}

El valor presente neto se define como el valor presente de los flujos de efectivo de entrada y de salida de un proyecto de inversión. Por tanto, el valor presente neto muestra el beneficio total obtenido de la inversión en un proyecto, el cual se calcula utilizando la siguiente ecuación (Lee y Lee 2006):

$$
N P V=-C_{I}+\sum_{t=0}^{n} \frac{B_{t}}{(1+r)^{t}}-\sum_{t=0}^{n} \frac{C_{t}}{(1+r)^{t}}
$$

Donde $\mathrm{n}$ es el tiempo de vida del proyecto, $\mathrm{t}$ es el período de tiempo el cual se expresa en años, $1+r$ es la tasa real de descuento, $B_{t}$ son todos los ingresos del proyecto durante un año $t$, por ejemplo ingresos provenientes de la venta de electricidad, mientras que $C_{t}$ son los costos de administración. Para calcular la tasa de descuento real se emplea la siguiente ecuación:

$$
1+r=\frac{1+i}{1+s}
$$

Donde $i$ es la inflación y $s$ es la tasa de descuento nominal. La inversión inicial incluye el precio del 
aerogenerador, precio de la torre, transportación y costos de instalación. Se asume que el costo de transportación y el costo de instalación representan el $30 \%$ del precio del aerogenerador. Todos los otros gastos de inversión inicial como: cables, conexión a la red, son ignorados para simplificar los cálculos. Por otro lado, los gastos anualmente recurrentes son: los costos de operación y administración. El resultado sirve como criterio acerca de si el proyecto debe llevarse a cabo o no, si el valor presente neto es mayor a cero, indica que el proyecto debe llevarse a cabo, si el valor presente neto es cero se obtiene como rendimiento exactamente la tasa de descuento nominal (no se obtienen beneficios adicionales) y si el valor presente neto es menor a cero el proyecto no es rentable.

\section{Tasa Interna de Rendimiento}

Otro parámetro importante es la tasa interna de rendimiento (TIR) este se define como el valor de la tasa de descuento con la cual el valor presente neto se vuelve cero, es decir, cuando el valor presente de todos los costos es igual al valor presente de todos los ingresos; igualando la ecuación (9) a cero, obtenemos la ecuación:

$$
\sum_{t=0}^{n} \frac{B_{t}}{(1+I R R)^{t}}=C_{I}-\sum_{t=0}^{n} \frac{C_{t}}{(1+I R R)^{t}}
$$

Donde IRR es la tasa interna de rendimiento. Un proyecto se considera como factible si la tasa interna de rendimiento es mayor a la tasa de descuento.

\section{LCOE}

El costo nivelado de electricidad, es el valor presente neto del costo de producir una unidad de energía eléctrica, considerando el período de vida del proyecto. Este enfoque ha sido ampliamente usado para comparar tecnologías de producción de energía en términos de costo-eficiencia, sin embargo, esta herramienta, depende de muchos parámetros que solo son parcialmente conocidos o que implican algún grado de incertidumbre. El LCOE se obtiene de acuerdo a la siguiente ecuación (Sajid et al. 2017):

$$
L C O E=\frac{\sum_{t=1}^{n}\left(\frac{I_{t}+O M_{t}}{(1+r)^{t}}\right)}{\sum_{t=1}^{n} A E P_{t}}
$$

Donde $I_{t}$ Inversión en el año t, $O M_{t}$ son los costos de operación y mantenimiento, y $A E P_{i}$ es la producción anual de energía para el aerogenerador $i$.

\section{Período de recuperación}

El período de recuperación n, es el tiempo en el cual se espera recuperar la inversión inicial con los flujos de efectivo en los siguientes años. Se calcula usando la ecuación (13) (Lee y Lee 2006):

$$
n=\sum_{t=0} F C_{t}=0
$$

Donde $F C_{t}$ es el flujo de efectivo en el año t.

\section{Razón costo beneficio}

Finalmente, cuando se comparan dos proyectos con diferentes niveles de inversión inicial, juzgar su mérito solamente en base de su valor presente neto puede llevar a conclusiones equivocadas. Ya que el proyecto 
con la inversión inicial más alta tiende a mostrar un mejor resultado del valor presente neto que el proyecto con menores requerimientos de capital. Bajo tales circunstancias, la proporción costo-beneficio, es una mejor herramienta para juzgar la viabilidad financiera. La proporción costo-beneficio es la razón entre el valor presente acumulado de todos los beneficios con respecto al valor presente acumulado de todos los costos, incluyendo la inversión inicial se calcula de la siguiente manera:

$$
B C R=\frac{\sum_{t=0}^{n} \frac{B_{t}}{(1+r)^{t}}}{C_{I}+\sum_{t=0}^{n} \frac{C_{t}}{(1+r)^{t}}}
$$

El cálculo del valor presente neto, de la tasa interna de rendimiento, del período para recuperar la inversión, el LCOE y la tasa costo/beneficio fueron calculados bajo los siguientes supuestos:

a) El capital inicial I se calculó asumiendo un precio de 2102 dólares por kilowatt de capacidad del aerogenerador ${ }^{2}$.

b) Se consideró que la vida útil t del aerogenerador es de 20 años.

c) Se asume una tasa de interés nominal s del $10 \%$.

d) Una tasa de inflación inf del $6.7 \%$.

e) Los costos de operación y mantenimientos además de los costos de reparación se asumen como 32 dólares por kilowatt de capacidad del aerogenerador ${ }^{3}$.

\section{Resultados}

\subsection{Distribución de Weibull}

Los cálculos de la media y de la función de distribución de la probabilidad de la velocidad del viento fueron realizados para la región de Apan, Hidalgo. Se recolectaron y analizaron datos de la velocidad del viento en el período 2015 a 2017. La media anual del viento su dirección, la frecuencia y los parámetros c y $\mathrm{k}$ se presentan en el cuadro 1.

Cuadro 1. Distribución de Weibull.

\begin{tabular}{|c|c|c|c|c|}
\hline & $\begin{array}{c}c \\
\mathrm{~m} / \mathrm{s}\end{array}$ & $\begin{array}{l}k \\
-\end{array}$ & $\begin{array}{c}\text { Velocidad del viento } \\
\mathrm{m} / \mathrm{s}\end{array}$ & $\begin{array}{c}\text { Frecuencia } \\
\%\end{array}$ \\
\hline $\mathrm{N}$ & 5.991 & 3.383 & 5.381 & 6.626 \\
\hline NNE & 5.263 & 2.189 & 4.661 & 31.312 \\
\hline ENE & 3.161 & 1.727 & 2.817 & 6.203 \\
\hline $\mathrm{E}$ & 2.486 & 1.597 & 2.229 & 6.412 \\
\hline ESE & 3.037 & 1.916 & 2.694 & 12.505 \\
\hline SSE & 2.973 & 1.498 & 2.684 & 10.988 \\
\hline $\mathrm{S}$ & 2.15 & 1.333 & 1.976 & 8.057 \\
\hline $\mathrm{SSO}$ & 1.888 & 1.35 & 1.731 & 6.869 \\
\hline OSO & 2.286 & 1.297 & 2.112 & 6.643 \\
\hline $\mathrm{O}$ & 2.979 & 1.643 & 2.665 & 3.365 \\
\hline $\mathrm{ONO}$ & 2.545 & 1.759 & 2.266 & 0.834 \\
\hline $\mathrm{NNO}$ & 2.211 & 1.78 & 1.967 & 0.185 \\
\hline Todas las direcciones & 3.667 & 1.561 & 3.296 & 100 \\
\hline
\end{tabular}

\footnotetext{
${ }^{2} \mathrm{El}$ costo de 2102 dólares/kW está basado en los datos de costo promedio de instalación de nuevas granjas eólicas en países seleccionados de la OCDE para México, IRENA (2015)

${ }^{3} \mathrm{El}$ valor de 32 dólares es el valor redondeado del $1.5 \%$ del capital inicial, este porcentaje es $.5 \%$ mayor del porcentaje que se considera en la descomposición de costos de capital para una granja eólica terrestre de 20MW en México, IRENA (2015)
} 
Los estimados de la producción de potencia están basados en el parámetro de forma k y el parámetro de escala c. $\mathrm{k}$ determina la uniformidad del viento. El parámetro de forma $\mathrm{k}$, puede ser interpretado directamente como sigue: un valor de $k<1$ indica que hay mayor concentración de energía en el viento por debajo de la velocidad promedio. Un valor de $k=1$ indica que la velocidad del viento es constante. Un valor de $k>1$ indica que la velocidad del viento está dominada por valores por encima de la velocidad media. Los valores obtenidos para el parámetro $\mathrm{k}$ son mayores a 1 lo que indica que la velocidad del viento en la locación estudiada está dominada por valores por encima de la velocidad media. La velocidad media del viento en el período de estudio es de $3.292 \mathrm{~m} / \mathrm{s}$. Los parámetros $k$ y $c$ tienen una media de 1.561 y 3.667 respectivamente en el período de estudio.

Empleando los parámetros obtenidos del cálculo de la distribución Weibull podemos calcular la velocidad del viento más probable y el viento que produce la mayor cantidad de energía. Los resultados son: el viento más probable es de $1.83 \mathrm{~m} / \mathrm{s}$ y la velocidad del viento que conlleva la máxima energía es de $6.25 \mathrm{~m} / \mathrm{s}$.

\section{Velocidad del viento a diferentes alturas}

Para poder calcular la variación de la velocidad del viento a diferentes alturas, donde se espera que trabajen los aerogeneradores, es necesario tomar en cuenta la aspereza y el tipo de terreno. El cuadro 2 muestra los resultados de la ecuación (1).

Cuadro 2. Velocidad del viento a diferentes alturas.

\begin{tabular}{|c|c|}
\hline Altura sobre el suelo & Velocidad del viento \\
\hline $150 \mathrm{~m}$ & $6.02 \mathrm{~m} / \mathrm{s}$ \\
\hline $140 \mathrm{~m}$ & $5.95 \mathrm{~m} / \mathrm{s}$ \\
\hline $130 \mathrm{~m}$ & $5.88 \mathrm{~m} / \mathrm{s}$ \\
\hline $120 \mathrm{~m}$ & $5.79 \mathrm{~m} / \mathrm{s}$ \\
\hline $110 \mathrm{~m}$ & $5.71 \mathrm{~m} / \mathrm{s}$ \\
\hline $100 \mathrm{~m}$ & $5.61 \mathrm{~m} / \mathrm{s}$ \\
\hline $90 \mathrm{~m}$ & $5.50 \mathrm{~m} / \mathrm{s}$ \\
\hline $80 \mathrm{~m}$ & $5.38 \mathrm{~m} / \mathrm{s}$ \\
\hline $70 \mathrm{~m}$ & $5.25 \mathrm{~m} / \mathrm{s}$ \\
\hline $60 \mathrm{~m}$ & $5.09 \mathrm{~m} / \mathrm{s}$ \\
\hline $50 \mathrm{~m}$ & $4.91 \mathrm{~m} / \mathrm{s}$ \\
\hline $40 \mathrm{~m}$ & $4.68 \mathrm{~m} / \mathrm{s}$ \\
\hline $30 \mathrm{~m}$ & $4.39 \mathrm{~m} / \mathrm{s}$ \\
\hline $20 \mathrm{~m}$ & $3.97 \mathrm{~m} / \mathrm{s}$ \\
\hline $10 \mathrm{~m}$ & $3.27 \mathrm{~m} / \mathrm{s}$ \\
\hline
\end{tabular}

Fuente: elaboración propia con datos del SMN

Los aerogeneradores modernos pueden alcanzar una altura de 166 metros. A partir de los 90 metros de altura se puede calificar el potencial del viento en Apan, Hidalgo como bueno de acuerdo a la clasificación de Ramachandra et al. (1997) la cual se puede observar en el cuadro 3.

Cuadro 3. Calidad del potencial del viento según su velocidad.

\begin{tabular}{|c|c|}
\hline Velocidad media anual del viento a $\mathbf{1 0}$ m de altura & Valor indicado del recurso del viento \\
\hline Debajo $4.5 \mathrm{~m} / \mathrm{s}$ & Pobre \\
\hline 4.5 a $5.4 \mathrm{~m} / \mathrm{s}$ & Marginal \\
\hline 5.4 a $6.7 \mathrm{~m} / \mathrm{s}$ & Bueno a muy bueno \\
\hline Por encima de $6.7 \mathrm{~m} / \mathrm{s}$ & Excepcional \\
\hline
\end{tabular}


Fuente: Ramachandra et al. (1997)

\section{Producción de energía anual y factor de capacidad}

Para poder evaluar el desempeño de la energía del viento en Apan, Hidalgo, se consideraron doce aerogeneradores con potencia declarada que va desde los 1700 kilowatts (kW) hasta los $4200 \mathrm{~kW}$. El objetivo es identificar el aerogenerador que tenga un mejor ajuste al régimen de viento del sitio en consideración, en este caso Apan, Hidalgo. Las especificaciones técnicas de estos aerogeneradores incluyen: el productor, el modelo la capacidad el diámetro del rotor, velocidad de inicio, velocidad de corte y velocidad declarada se muestran en el cuadro 4. Todos los aerogeneradores en consideración tienen alturas por encima de los diez metros por lo que es necesario utilizar la aproximación a la ley de poder para encontrar las diferentes velocidades del viento que correspondan a la altura del aerogenerador; los resultados de la aproximación de la ley de poder se pueden observar en el cuadro 2.

Cuadro 4. Especificaciones técnicas de los distintos aerogeneradores.

\begin{tabular}{|c|c|c|c|c|c|c|}
\hline Productor & Modelo & Capacidad & $\begin{array}{c}\text { Diametro } \\
\text { del Rotor }\end{array}$ & $\begin{array}{c}\text { Velocidad } \\
\text { de inicio }\end{array}$ & $\begin{array}{c}\text { Velocidad } \\
\text { de corte }\end{array}$ & $\begin{array}{c}\text { Velocidad } \\
\text { declarada }\end{array}$ \\
\hline GE Wind Energy & GE $107 / 103$ & $1700 \mathrm{~kW}$ & $103 \mathrm{~m}$. & $3 \mathrm{~m} / \mathrm{s}$ & $23 \mathrm{~m} / \mathrm{s}$ & $10 \mathrm{~m} / \mathrm{s}$ \\
\hline Vestas & V100-2.0 & $1800 \mathrm{~kW}$ & $100 \mathrm{~m}$. & $3 \mathrm{~m} / \mathrm{s}$ & $22 \mathrm{~m} / \mathrm{s}$ & $12 \mathrm{~m} / \mathrm{s}$ \\
\hline Vestas & V110-2.0 & $2000 \mathrm{~kW}$ & $100 \mathrm{~m}$. & $3 \mathrm{~m} / \mathrm{s}$ & $20 \mathrm{~m} / \mathrm{s}$ & $11.5 \mathrm{~m} / \mathrm{s}$ \\
\hline Nordex & N117 & $2400 \mathrm{~kW}$ & $117 \mathrm{~m}$. & $3 \mathrm{~m} / \mathrm{s}$ & $20 \mathrm{~m} / \mathrm{s}$ & $11 \mathrm{~m} / \mathrm{s}$ \\
\hline Gamesa & G114 & $2500 \mathrm{~kW}$ & $114 \mathrm{~m}$. & $2 \mathrm{~m} / \mathrm{s}$ & $25 \mathrm{~m} / \mathrm{s}$ & $10 \mathrm{~m} / \mathrm{s}$ \\
\hline GE Wind Energy & GE $2.75 / 120$ & $2750 \mathrm{~kW}$ & $120 \mathrm{~m}$. & $3.5 \mathrm{~m} / \mathrm{s}$ & $25 \mathrm{~m} / \mathrm{s}$ & $13 \mathrm{~m} / \mathrm{s}$ \\
\hline Senvion & $3.0 M 122$ & $3000 \mathrm{~kW}$ & $122 \mathrm{~m}$. & $3 \mathrm{~m} / \mathrm{s}$ & $22 \mathrm{~m} / \mathrm{s}$ & $11.5 \mathrm{~m} / \mathrm{s}$ \\
\hline Vensys & 120 & $3000 \mathrm{~kW}$ & $120 \mathrm{~m}$. & $3 \mathrm{~m} / \mathrm{s}$ & $22 \mathrm{~m} / \mathrm{s}$ & \\
\hline Vestas & V126 & $3000 \mathrm{~kW}$ & $126 \mathrm{~m}$. & $3 \mathrm{~m} / \mathrm{s}$ & $22.5 \mathrm{~m} / \mathrm{s}$ & $12 \mathrm{~m} / \mathrm{s}$ \\
\hline Nordex & N131 & $3000 \mathrm{~kW}$ & $131 \mathrm{~m}$. & $3 \mathrm{~m} / \mathrm{s}$ & $20 \mathrm{~m} / \mathrm{s}$ & $11.5 \mathrm{~m} / \mathrm{s}$ \\
\hline Enercon & E-115 & $3000 \mathrm{~kW}$ & $115.7 \mathrm{~m}$. & $2 \mathrm{~m} / \mathrm{s}$ & $25 \mathrm{~m} / \mathrm{s}$ & $11.5 \mathrm{~m} / \mathrm{s}$ \\
\hline Enercon & E-126 EP4 & $4200 \mathrm{~kW}$ & $126 \mathrm{~m}$. & $3 \mathrm{~m} / \mathrm{s}$ & $34 \mathrm{~m} / \mathrm{s}$ & $13.5 \mathrm{~m} / \mathrm{s}$ \\
\hline
\end{tabular}

Fuente: elaboración propia con datos del fabricante

La estimación del factor de capacidad, la energía generada anualmente por cada modelo y la altura del aerogenerador se muestra en el cuadro 5 .

Cuadro 5. Producción de energía, factor de capacidad y altura de la aerogenerador.

\begin{tabular}{|c|c|c|c|}
\hline Tipo & $\begin{array}{c}\text { Producción } \\
\text { de energía }\end{array}$ & $\begin{array}{c}\text { Factor de } \\
\text { capacidad }\end{array}$ & $\begin{array}{c}\text { Altura del } \\
\text { aerogenerador }\end{array}$ \\
\hline GE $107 / 103$ & $4130 \mathrm{MW} / \mathrm{h} / \mathrm{a}$ & $27.7 \%$ & $96 \mathrm{~m}$. \\
\hline $\mathrm{V} 100-2.0$ & $3799 \mathrm{MW} / \mathrm{h} / \mathrm{a}$ & $24 \%$ & $95 \mathrm{~m}$. \\
\hline $\mathrm{V} 110-2.0$ & $4917 \mathrm{MW} / \mathrm{h} / \mathrm{a}$ & $28 \%$ & $125 \mathrm{~m}$. \\
\hline $\mathrm{N} 117$ & $5788 \mathrm{MW} / \mathrm{h} / \mathrm{a}$ & $27.5 \%$ & $140 \mathrm{~m}$. \\
\hline $\mathrm{G} 114$ & $5695 \mathrm{MW} / \mathrm{h} / \mathrm{a}$ & $26 \%$ & $140 \mathrm{~m}$. \\
\hline $\mathrm{GE} 2.75 / 120$ & $6377 \mathrm{MW} / \mathrm{h} / \mathrm{a}$ & $26.5 \%$ & $139 \mathrm{~m}$. \\
\hline $3.0 \mathrm{M} 122$ & $6598 \mathrm{MW} / \mathrm{h} / \mathrm{a}$ & $25.1 \%$ & $139 \mathrm{~m}$. \\
\hline 120 & $6223 \mathrm{MW} / \mathrm{h} / \mathrm{a}$ & $23.7 \%$ & $140 \mathrm{~m}$. \\
\hline $\mathrm{V} 126$ & $6988 \mathrm{MW} / \mathrm{h} / \mathrm{a}$ & $26.6 \%$ & $166 \mathrm{~m}$. \\
\hline $\mathrm{N} 131$ & $6870 \mathrm{MW} / \mathrm{h} / \mathrm{a}$ & $26.1 \%$ & $114 \mathrm{~m}$. \\
\hline $\mathrm{E}-115$ & $6387 \mathrm{MW} / \mathrm{h} / \mathrm{a}$ & $24.3 \%$ & $149 \mathrm{~m}$. \\
\hline $\mathrm{E}-126 \mathrm{EP} 4$ & $7992 \mathrm{MW} / \mathrm{h} / \mathrm{a}$ & $21.7 \%$ & $159 \mathrm{~m}$ \\
\hline
\end{tabular}

Fuente: elaboración propia 
La altura a la cual se puede instalar un aerogenerador afecta la velocidad del viento que debemos considerar. El calculo de la producción de energía cuyos resultados se encuentran en el cuadro 5 se realizó utilizando la velocidad del viento que corresponde a la altura máxima a la que se puede instalar dicho aerogenerador. El aerogenerador que se puede instalar a mayor altura es el V126, la altura de instalación es de 166 metros. El aerogenerador con la altura de instalación más baja es el V100-2.0 con una altura de instalación de 95 metros.

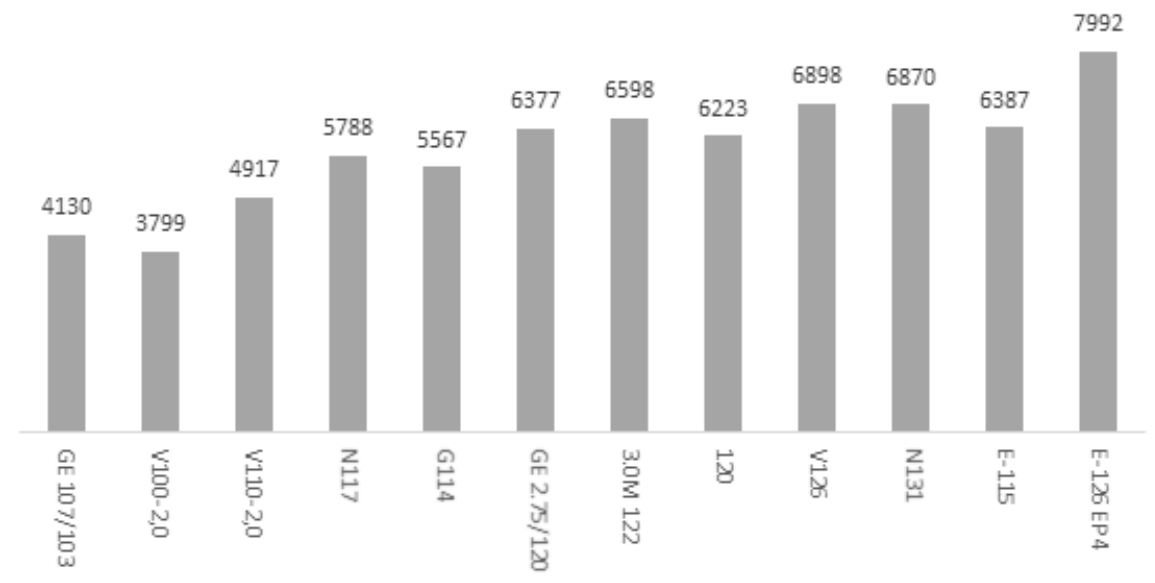

Gráfica 1. Producción anual de energía por aerogenerador.

(Megawatts)

Fuente: elaboración propia

El aerogenerador con la mayor producción de energía anual es la E-126 EP4 con una producción anual de 7992 MW de energía. El segundo aerogenerador con mayor producción es el V126 con una producción anual de $6898 \mathrm{MW}$ anuales. El siguiente aerogenerador con mayor producción es el N131 con una producción anual de $6870 \mathrm{MW}$. Cabe destacar que el aerogenerador E-126 EP4 tiene una capacidad de $4200 \mathrm{~kW}$ y los aerogeneradores V126 y N131 tienen capacidad de $3000 \mathrm{~kW}$. En cuanto a los aerogeneradores de capacidad de $2400 \mathrm{~kW}$ a $2750 \mathrm{~kW}$ el aerogenerdos con mayor producción es el GE 2.75/120 con una producción de 6377 MW. Y para los aerogeneradores de $1700 \mathrm{~kW}$ a $2000 \mathrm{~kW}$ el aerogenerador con mayor producción es el V110-2.0 con una producción de 4917 MW anuales.

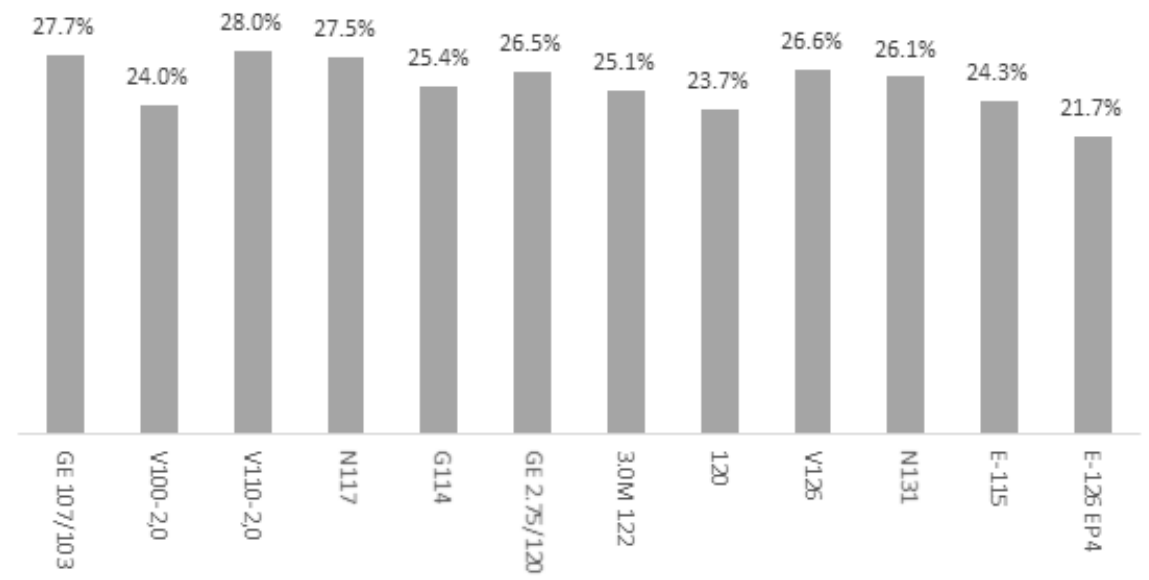

Gráfica 2. Factor de capacidad por aerogenerador.

Fuente: elaboración propia

El aerogenerador con mayor factor de capacidad es el V110-2.0 con un factor de capacidad de $28 \%$. 
El aerogenerador con el segundo mayor factor de capacidad es el GE 107/103 de 27.7\%. Mientras que el aerognerador E-126 EP4 es la que tiene el menor factor de capacidad con un valor de $21.7 \%$. Para una inversión costo efectiva en la potencia del viento, se recomienda que el factor de capacidad debe ser mayor a $25 \%$ de acuerdo a Chauhan y Saini (2014). Basado en esto, se encuentra que los modelos de aerogeneradores que no alcanzan un $25 \%$ de factor de capacidad son los siguientes: Vestas V100-2.0, Vensys 120, Enercon E-115 y Enercon E-126 EP4. El resto de los aerogeneradores tienen factores de capacidad por encima del $25 \%$ lo que indica que tienen un buen ajuste para las características del viento que encontramos en Apan, Hidalgo.

\subsection{Análisis financiero}

Los resultados muestran que de acuerdo a los parámetros económicos Valor Presente Neto y razón costo beneficio, los aerogeneradores económicamente viables son: GE 107/103, V110-2.0, N117, GE 2.75/120, V126 y N131. El aerogenerador con la tasa interna de rendimiento más alta es V110-2,0 con un valor de 4.1\%, mientras que el aerogenerador con la tasa interna de rendimiento más baja es la E-126 EP4 con una tasa de $.7 \%$. Por lo tanto, ningún aerogenerador tiene un rendimiento mayor a la tasa de descuento y no se puede recomendar su instalación.

Por otra parte, el aerogenerador con el LCOE más bajo es el N117 con un valor de $\$ .039$ centavos por kilowatt. El período más corto para recuperar la inversión inicial corresponde al aerogenerador V110-2.0 con un período de recuperación de 17.7 años, mientras que el período más largo es del aerogenerador E-126 EP4 con un período de 27.9 años.

Cuadro 6. Resultados del análisis económico.

\begin{tabular}{|c|c|c|c|c|c|}
\hline Modelo & $\begin{array}{c}\text { Valor Presente Neto } \\
\text { (Dólares estadounidenses) }\end{array}$ & $\begin{array}{l}\text { Tasa interna de } \\
\text { rendimiento }(\%)\end{array}$ & $\begin{array}{l}\text { Períodos para } \\
\text { recuperar la } \\
\text { inversión (años) }\end{array}$ & $\begin{array}{c}\text { LCOE } \\
\text { centavos/kilowatt-hora } \\
\text { (Dólares estadounidenses) }\end{array}$ & $\begin{array}{l}\text { Tasa de costo } \\
\text { beneficio (\%) }\end{array}$ \\
\hline GE $107 / 103$ & $\$ 282767$ & $3.9 \%$ & 18 & $\$ .045$ & 1.06 \\
\hline V100-2,0 & $-\$ 348000$ & $2.1 \%$ & 22.9 & $\$ .051$ & 0.92 \\
\hline V110-2,0 & $\$ 398289$ & $4.1 \%$ & 17.7 & $\$ .041$ & 1.08 \\
\hline N117 & $\$ 351161$ & $3.8 \%$ & 18.3 & $\$ .039$ & 1.06 \\
\hline G114 & $-\$ 11144$ & $3.1 \%$ & 20.1 & $\$ .044$ & 0.99 \\
\hline GE $2.75 / 120$ & $\$ 114640$ & $3.3 \%$ & 19.5 & $\$ .044$ & 1.02 \\
\hline $3.0 \mathrm{M} 122$ & $-\$ 279579$ & $2.6 \%$ & 21.3 & $\$ .046$ & 0.96 \\
\hline 120 & $-\$ 702575$ & $1.8 \%$ & 23.6 & $\$ .049$ & 0.91 \\
\hline V126 & $\$ 160338$ & $3.4 \%$ & 19.3 & $\$ .043$ & 1.02 \\
\hline N131 & $\$ 27235$ & $3.1 \%$ & 19.9 & $\$ .044$ & 1.003 \\
\hline E-115 & $-\$ 517585$ & $2.1 \%$ & 22.6 & $\$ .047$ & 0.93 \\
\hline E-126 EP4 & $-\$ 1795984$ & $.7 \%$ & 27.9 & $\$ .054$ & 0.83 \\
\hline
\end{tabular}

El resultado del análisis económico indica que el aerogenerador V110-2.0 es el que tiene mejor ajuste a las características del lugar estudiado, seguido muy de cerca por el aerogenerador N117. Los resultados se muestran en el cuadro 6.

\section{Conclusiones}

El presente estudio se enfoca en el análisis preliminar del potencial energetico del viento para Apan, Hidalgo. Se estudió el perfil del viento en el período de dos años a la altura de 10 metros del anemometro, para analizar la conveniencia de la instalación de aerogeneradores en la locación para la generación de electricidad. Los resultados clave del estudio son: 
- Los parámetros de la velocidad del viento c y k son 3.667 y 1.561.

- Ocho de doce modelos de aerogeneradores considerados tienen factores de capacidad por encima de $25 \%$. El modelo de aerogenerador con el mayor factor de capacidad es la Vestas V110-2.0 y el modelo con el factor de capacidad más bajo es la Enercon E-126 EP4.

- Se estudiaron doce modelos diferentes de aerogeneradores, de los cuales solo seis son económicamente viables (tienen Valor Presente Neto positivo) estos son: GE 107/103, V110-2.0, N117, GE 2.75/120, V126 y N131.

- El costo nivelado de la energía varía en un rango desde $\$ .039$ centavos kilowatt/hora hasta $\$ .054$ centavos kilowatt/hora. El aerogenerador con el costo de energía más bajo es el modelo N117 y el modelo con el costo más alto es el E-126 EP4.

- Mientras que el aerogenerador con los mejores resultados en los parámetros financieros: tasa interna de rendimiento, valor presente neto, período de recuperación y razón costo beneficio es el aerogenerador modelo V110-2.0. Así mismo el modelo V110-2.0 es también el modelo que mejores resultados obtiene de acuerdo al análisis técnico. Sin embargo, cabe apuntar que ningún aerogenerador tiene una tasa interna de rendimiento mayor a la tasa de descuento por lo que no se puede recomendar la instalación de un aerogenerador en Apan, Hgo.

- Los datos sugieren que el potencial del viento en Apan, Hidalgo es adecuado para aplicaciones que no están conectadas a la red eléctrica e insuficiente para la producción de energía a escala comercial.

\section{Referencias}

[1] Aien, M., M. Rashidinejad, y M. Fotuhi-Firuzabad (2014) On possibilistic and probabilistic uncertainty assessment of power flow problem: a review and a new approach. Renewable and Sustainable Energy Reviews, 37, pp. 883-895. doi:10.1016/j.rser.2014.05.063

[2] Akpinar, A. (2013) Evaluation of wind energy potentiality at coastal locations along the north eastern coasts of Turkey. Energy, 50, pp. 395-405. doi:10.1016/j.energy.2012.11.019

[3] Alamdari, P., O. Nematollahi, y M. Mirhosseini (2012) Assessment of wind energy in Iran: a review. Renewable and Sustainable Energy Reviews, 16, pp. 836-860. doi:10.1016/j.rser.2011.09.007

[4] AMDEE (2018) Asociación Mexicana de Energía Eólica Recuperado diciembre 15, 2017, de https://www.amdee.org/capacidad-instalada

[5] BP (2017). BP statistical review of world energy. Recuperado diciembre 5, 2017, de: https://www.bp.com/content/dam/bp/pdf/energy-economics/energy-outlook-2017/bp-energy-outlook-2017.pdf

[6] Cancino-Solorzano, Y., A. J. Gutiérrez-Trashorras, y J. Xiberta-Bernat, (2011) Current state of wind energy in Mexico, achievements and perspectives. Renewable and Sustainable Energy Reviews, 15, pp. 3352-3557. doi:10.1016/j.rser.2011.05.009

[7] Chauhan, A., y R. P. Saini, (2014) Statistical analysis of wind speed data using Weibull distribution parameters. 1st International Conference on Non Conventional Energy, Kolyani, Bengala Occidental, India. doi:10.1109/iconce.2014.6808712

[8] Dabbaghiyan, A., F. Fazelpour, M. Dehghan, y M. A. Rosen, (2016) Evaluation of wind energy potential in province of Bushehr, Iran. Renewable and Sustainable Energy Reviews, 55, pp. 455-456. doi:10.1016/j.rser.2015.10.148

[9] EIA (2016) International Energy Outlook. Recuperado diciembre $5, \quad 2017 \quad$ de https://www.eia.gov/outlooks/aeo/pdf/0383(2016).pdf 
[10] Ellabban, O., H. Abu-Rub, y F. Blaabjerg, (2014) Renewable energy resources: Current status, future prospects and their enabling technology. Renewable and Sustainable Energy Reviews. 39, pp. 748-764. doi:10.1016/j.rser.2014.07.113

[11] Fazelpour, F., E. Markarian, y N. Soltani, (2017) Wind energy potential and economic assessment of four locations in Sistan and Balouchestan province in Iran. Renewable Energy, 108, pp. 646-667. doi:10.1016/j.renene.2017.03.072

[12] Hernández-Escobedo, Q., F. Manzano-Agugliaro, A. Gazquez-Parra, y A. Zapata-Sierra, (2011) Is the wind a periodical phenomenon? The case of Mexico. Renewable and Sustainable Energy Reviews, 15, pp. 721-728. doi:10.1016/j.rser.2010.09.023

[13] Hernández-Escobedo, Q., F. Manzano-Agugliaro, y A. Zapata-Sierra, (2010) The wind power of Mexico. Renewable and Sustainable Energy Reviews, 14, pp. 2830-2840. doi:10.1016/j.rser.2010.07.019

[14] Hernández-Escobedo, Q., R. Saldaña-Flores, E. R. Rodríguez-García, y F. Manzano-Agugliaro, (2014) Wind energy resource in Northern Mexico. Renewable and Sustainable Energy Reviews, 14, pp. 2830-2840. doi:10.1016/j.rser.2014.01.043

[15] IRENA (2015), Renewable Power Generation Costs in 2014.

[16] Lee, C. F y A. C. Lee, (2006). Encyclopedia of Finance. United States, Springer Science Business Media.

[17] Manwell, J.F., J.G McGowan, y A.L. Rogers (2002) Wind energy explained: theory, design and application. Amherst, USA: John Wiley Sons,

[18] Magar, V., M. S. Gross, y L. González-García, (2018) Offshore wind energy resource assesment under techno-economic and social-ecological constrains. Ocean and Coastal Management, 152, pp. 77-87. doi:10.1016/j.ocecoaman.2017.10.007

[19] Mohammadi, K. y A. Mostafaeipour (2013), Economic feasibility of developing wind turbines in Aligoodarz, Iran. Energy Conversion Management. 76, pp. 645-653. doi:10.1016/j.enconman.2013.06.053

[20] Pérez-Denicia, E., F. Fernández-Luqueño, D. Vilariño-Ayala, L. M. Montaño-Zetina, y L. A. Maldonado López (2017) Renewable energy sources for electricity generation in Mexico: A review. Renewable and Sustainable Energy Reviews, 78, pp. 597-613. doi:10.1016/j.rser.2017.05.009

[21] Ramachandra, T., Subramanian, D., y Joshi, N. (1997) Wind Energy Potential Assessment in Uttara Kanada, District of Karnataka. Renewable Energy, 10 (4), pp. 585-611. doi:10.1016/s0960-1481(96)00034-1

[22] Sajid, A., L. Sang-Moon, y J. Choon-Man, (2017) Techno-Economic Assessment of Wind Energy Potential at Three Locations in South Korea Using Long-Term Measured Wind Data. Energies, 10 (9), pp. 1442. doi:10.3390/en10091442

[23] SENER (2015) Prospectivas del sector eléctrico 2015-2029. México. Secretaría de Energía.

[24] Stevens, M.J.M y P.T Smulders. (1979) Estimation of the parameters of the Weibull wind speed distribution for wind energy utilization purposes, Wind Energy, 3 (2), pp. 132-145.

[25] Wallace, J. M. y P. V. Hobs, (2006) Atmospheric Science; An Introductory Survey. Elsevier. Second Edition. doi:10.1016/b978-0-12-732951-2.50012-0 